\title{
Novel Predicted B-Cell Epitopes of PSMA for Development of Prostate Cancer Vaccine
}

\author{
Pejman Hashemzadeh ${ }^{1}$ ([) $\cdot$ Vajihe Ghorbanzadeh $^{2}$ (C) . Seyede Maryam Valizadeh Otaghsara ${ }^{1}$ (i) \\ Hassan Dariushnejad ${ }^{1}$ (1)
}

Accepted: 26 October 2019 / Published online: 4 November 2019

(c) Springer Nature B.V. 2019

\begin{abstract}
Prostate cancer is one of the most common cancers around the world. Vaccines are a new hope for prevention of this cancer. In the case of prostate cancer, PSMA is considered as a target for vaccine development. Here, the initial data from the computational analysis of this specific antigen of the prostate membrane to find potential B-cell epitopes are described using a new bioinformatic tools. Based on the results, 673RHVIYAPSSHNKYAGE25 is the peptide with best binding affinity. These data may be useful for the effective vaccines development.
\end{abstract}

Keywords Prostate cancer · B-cell · Epitope $\cdot$ PSMA

\section{Introduction}

Prostate is a small gland located below the men's bladder and helps sperm production. Testosterone hormones regulate the function of the prostate gland (Gathirua-Mwangi and Zhang 2014). Prostate cancer is the most common cancer among men; about one in six man (16\%) (Jemal et al. 2005). Prostate cancer in the populations of Africa and Asia has the highest and lowest incidence rates, respectively (Steinberg et al. 1990). Researchers believe that the incidence of prostate cancer is related to hormonal factors. For example, the conversion of testosterone to dihydrotestosterone is known as an important factor in the progression of this disease (Parsons et al. 2005). Development of prostate cancer is associated with several factors including age, diet, heredity, race

Hassan Dariushnejad

dariushnejad@gmail.com

Pejman Hashemzadeh

Pejman7genetian@gmail.com

Vajihe Ghorbanzadeh

vghorbanzadeh@gmail.com

Seyede Maryam Valizadeh Otaghsara

maryamvallizade@gmail.com

1 Department of Medical Biotechnology, Faculty of Medicine, Lorestan University of Medical Sciences, Khorramabad, Iran

2 Razi Herbal Medicines Research Center, Lorestan University of Medical Sciences, Khorramabad, Iran and the environment factor (Karan et al. 2008). The most important factor in developing prostate cancer among these factors is the rise in age (De Marzo 2008). The most common symptoms of prostate cancer are frequent and difficult urination, impotence in the urination, intermittent and poorly flowed urine, blood in the urine and seminal outflow with pain are main symptoms (Arrighi et al. 1990).

Prostate surgery, radiotherapy, chemotherapy, and hormone therapy are rutin treatments for this cancer (Stamey et al. 1993). Prostate cancer has poor prognosis and in the advanced stages the chance of treatment highly decreased (Voeks et al. 2002). Therefore, finding the more effective treatment for coping with this disease is very important (McNeel and Malkovsky 2005).

Immunological approaches have introduced new insights to treatment and prevention of this disease. Recombinant vaccine is one of this approaches. In this method, cancerous antigens or tissue that was targeted by $\mathrm{T}$ cells or B cells are used in the design of recombinant vaccines. (Hurwitz et al. 2003).

Prostatic membrane antigen (PSMA) is important antigen in prostate cancer and expression of it significantly increased (Jacobson 2007). PSMA has molecular weight about $100 \mathrm{kDa}$ and belong to membrane glycoprotein family (Zeng et al. 2005). Although this antigen express highly in prostate cancer tissue, its expression is limited in other tissues (Silver et al. 1997). For this reasons, PSMA 
Table 1 Predicted B-cells epitope

\begin{tabular}{llll}
\hline Rank & Sequence & Start position & Score \\
\hline 1 & RHVIYAPSSHNKYAGE & 673 & 0.96 \\
1 & LNGAGDPLTPGYPANE & 246 & 0.96 \\
2 & DSSIEGNYTLRVDCAP & 438 & 0.95 \\
3 & HSVYETYELVEKFYDP & 538 & 0.92 \\
4 & PGIYDALFDIESKVDP & 691 & 0.91 \\
4 & GWFIKSSNEATSITPK & 25 & 0.91 \\
5 & IASGRARYTKNWETNK & 515 & 0.90 \\
5 & TQKVKMHIHSTNEVTR & 324 & 0.90 \\
5 & IVIARYGKVFRGNKVK & 185 & 0.90 \\
\hline
\end{tabular}

is appropriate target for the development of cancer vaccine to prevent prostate cancer (Olson et al. 2007).

Based on many advances in bioinformatics, it has become a new alternative to the development of vaccines (Brusic et al. 2005). By increasing the amount of available information in the genome databases, vaccine specialists use epitope mapping tools to display vaccine candidates. New databases have been launched to facilitate epitope prediction (De Groot 2006).

The main objective of this study is to investigate the potential B cell epitope of PSMA antigen by using a new bioinformatics tools.

\section{Materials and Methods}

The complete sequence of PSMA antigen (Entry: A4UU13) retrived from the UniProt Knowledgebase (UniProtKB) in FASTA format. ABCpred Prediction Server was used to find potential B-cell epitopes (https://crdd. osdd.net/raghava/abcpred/). The ABCpred server provide a powerful tool for prediction of B cell epitope(s) in an antigen sequence by using an artificial neural network. ABCpred is the first server developed based on a neural network based technique (a machine-based technique) using fixed-length patterns (Saha and Raghava 2006).

\section{Results}

The ABCpred server predict approximately 81 epitope peptides. The peptides with the first 9 best orders of predicted binding affinities are presented in Table 1 .

\section{Discussion}

Prostate cancer is the second most common cancer and the sixth cause of cancer-related deaths in men (Ryan et al. 2013). Also, it is the most common cancer in men in developed countries (Drake 2010). Radiotherapy, chemotherapy, prostate surgery, hormonal therapy have been used for the treatment of prostate cancer. Most of these procedures have side effects and, in the case of advanced prostate cancer, these therapies are no longer effective (Tran et al. 2009; Zhou and Zhong 2004).

In recent years designing vaccines against cancers have been noticed. Lack of side effects, easy manufacturing, low cost, and immune system stimulation make vaccines ideal for cancers. PSMA is one of the prominent antigens in prostate cancer that can stimulate the immune system and produce antibodies (Zhu et al. 1999). PSMA expression in normal prostate tissue is less than prostate cancer (Olson et al. 2007). PSMA expression is restricted to the prostate secretory epithelium (Chang et al. 1999). Based on research, recombinant immunogen peptide that stimulates B-cell might have applications in the prevention of PSMA overexpressing prostate cancer (Dakappagari et al. 2000). Overexpression of PSMA in prostate cancer and its membranous nature have made this antigen as an effective candidate for the prevention and treatment of prostate cancer. Studies showed that PSMA based vaccine had no side effect in patients (Olson et al. 2007).

Although using PSMA antigen for vaccine had their beneficial but designing a multi-epitope vaccine will improve the efficiency of vaccine. Selecting appropriate epitopes with highest antigenicity for B cells is key point to develop of this kind of vaccines.

In this study, a new bioinformatic tool was used to predict potential B-cell epitopes. The determined peptides should be useful for further vaccine development because they can reduce the time and minimize the total number of required tests to find the possible proper epitopes.

\section{Conclusion}

A computational method was used to determine the potential B-cell epitopes of PSMA. According to this work, 673RHVIYAPSSHNKYAGE25 is the peptide with the best binding affinity. The results are only predictions and further confirmation is required. The peptide synthesis in the laboratory and the in vivo experimental study to test the efficacy are the next steps in the prostate cancer vaccine development. 


\section{Compliance with Ethical Standards}

Conflict of interest All authors declare that they have no conflict of interest.

Ethical Approval This article does not contain any studies with human participants or animals performed by any of the authors.

\section{References}

Arrighi H, Guess H, Metter E, Fozard J (1990) Symptoms and signs of prostatism as risk factors for prostatectomy. Prostate 16:253-261

Brusic V, August JT, Petrovsky N (2005) Information technologies for vaccine research. Expert Rev Vaccines 4:407-417. https://doi. org/10.1586/14760584.4.3.407

Chang SS, O'Keefe DS, Bacich DJ, Reuter VE, Heston WD, Gaudin PB (1999) Prostate-specific membrane antigen is produced in tumor-associated neovasculature. Clin Cancer Res 5:2674-2681

Dakappagari NK, Douglas DB, Triozzi PL, Stevens VC, Kaumaya PT (2000) Prevention of mammary tumors with a chimeric HER-2 B-cell epitope peptide vaccine. Cancer Res 60:3782-3789

De Groot AS (2006) Immunomics: discovering new targets for vaccines and therapeutics. Drug Discov Today 11:203-209. https:// doi.org/10.1016/s1359-6446(05)03720-7

De Marzo AM (2008) Interaction between dietary factors and inflammation in prostate carcinogenesis. The Johns Hopkins University School of Medicine, Baltimore, MD

Drake CG (2010) Prostate cancer as a model for tumour immunotherapy. Nat Rev Immunol 10:580

Gathirua-Mwangi WG, Zhang J (2014) Dietary factors and risk for advanced prostate cancer. Eur J Cancer Prev 23:96-109. https:// doi.org/10.1097/CEJ.0b013e3283647394

Hurwitz AA, Yanover P, Markowitz M, Allison JP, Kwon ED (2003) Prostate cancer. BioDrugs 17:131-138

Jacobson T (2007) Development and evaluation of a phage-display based vaccine against prostate cancer. Numerisk analys och datalogi, Kungliga Tekniska högskolan, Stockholm

Jemal A et al. (2005) Cancer statistics, 2005. CA: A Cancer J Clin 55:10-30

Karan D, Thrasher J, Lubaroff D (2008) Prostate cancer: genes, environment, immunity and the use of immunotherapy. Prostate Cancer Prost Dis 11:230
McNeel DG, Malkovsky M (2005) Immune-based therapies for prostate cancer. Immunol Lett 96:3-9

Olson WC, Heston WD, Rajasekaran AK (2007) Clinical trials of cancer therapies targeting prostate-specific membrane antigen. Rev Recent Clin Trials 2:182-190

Parsons JK, Carter HB, Platz EA, Wright EJ, Landis P, Metter EJ (2005) Serum testosterone and the risk of prostate cancer: potential implications for testosterone therapy. Cancer Epidemiol Prev Biomark 14:2257-2260

Ryan CJ et al (2013) Abiraterone in metastatic prostate cancer without previous chemotherapy. New Engl J Med 368:138-148

Saha S, Raghava GP (2006) Prediction of continuous B-cell epitopes in an antigen using recurrent neural network. Proteins 65:40-48. https://doi.org/10.1002/prot.21078

Silver DA, Pellicer I, Fair WR, Heston W, Cordon-Cardo C (1997) Prostate-specific membrane antigen expression in normal and malignant human tissues. Clin Cancer Res 3:81-85

Stamey TA, Freiha FS, McNeal JE, Redwine EA, Whittemore AS, Schmid HP (1993) Localized prostate cancer. Relationship of tumor volume to clinical significance for treatment of prostate cancer. Cancer 71:933-938

Steinberg GD, Carter BS, Beaty TH, Childs B, Walsh PC (1990) Family history and the risk of prostate cancer. Prostate 17:337-347

Tran C et al (2009) Development of a second-generation antiandrogen for treatment of advanced prostate cancer. Science 324:787-790

Voeks DJ, Martiniello-Wilks R, Russell PJ (2002) Derivation of MPR and TRAMP models of prostate cancer and prostate cancer metastasis for evaluation of therapeutic strategies. Urol Oncol 3:111-118

Zeng $\mathrm{H}$ et al (2005) Construction of prostate-specific expressed recombinant plasmids with high transcriptional activity of prostatespecific membrane antigen (PSMA) promoter/enhancer. J Androl 26:215-221

Zhou J, Zhong Y (2004) Breast cancer immunotherapy. Cell Mol Immunol 1:247-255

Zhu ZY et al (1999) PSMA mimotope isolated from phage displayed peptide library can induce PSMA specific immune response. Cell Res 9:271

Publisher's Note Springer Nature remains neutral with regard to jurisdictional claims in published maps and institutional affiliations. 